\title{
¿Pueden los videojuegos cambiar el mundo? Una introducción a los serious games.
}

Salvador Gómez García (2014). [versión Amazon Kindle]. Universidad Internacional de La Rioja, España. 117 p. (estimación). Recuperado de: http://www.amazon.com

\section{Alfonso Boullón Sabín}

\section{(2) OpenEdition}

12 Journals

Edición electrónica

URL: http://journals.openedition.org/ctd/2082

DOI: $10.4000 /$ ctd.2082

ISSN: 2491-1437

Editor

Chaire Unesco Pratiques émergentes en technologies et communication pour le développement

Edición impresa

Fecha de publicación: 12 noviembre 2015

\section{Referencia electrónica}

Alfonso Boullón Sabín, « ¿Pueden los videojuegos cambiar el mundo? Una introducción a los serious games. ", Communication, technologies et développement [En línea], 2 | 2015, Publicado el 12 junio 2019, consultado el 22 julio 2020. URL : http://journals.openedition.org/ctd/2082 ; DOI : https://doi.org/ $10.4000 /$ ctd.2082

Este documento fue generado automáticamente el 22 julio 2020.

Communication, technologies et développement 


\section{¿Pueden los videojuegos cambiar el mundo? Una introducción a los serious games.}

Salvador Gómez García (2014). [versión Amazon Kindle]. Universidad Internacional de La Rioja, España. 117 p. (estimación). Recuperado de: http://www.amazon.com

\section{Alfonso Boullón Sabín}

1 La progresiva popularización que han experimentado los videojuegos desde la década de los setenta del pasado siglo ha hecho que en la actualidad éstos sean considerados un importante medio de entretenimiento y de comunicación de masas de la industria cultural. Su capacidad de influencia en la generación de tendencias e iconos sociales puede ir más allá de la dimensión lúdica, y es en esa vertiente en la que se centra el libro aquí reseñado.

2 La obra, estructurada en seis capítulos, se presenta como una aproximación introductoria al amplio fenómeno de los videojuegos no vinculados al entretenimiento ; a aquellos que están orientados, en sus objetivos comunicativos, hacia la información y la persuasión : los serious games. El autor subraya, no obstante, que lo serio no debe presentarse en oposición a lo entretenido.

El marco teórico ocupa el primer capítulo. En un primer apartado, se analizan algunas de las aproximaciones clásicas más importantes a la hora de abordar el estudio de los juegos, los planteamientos iniciales en el estudio de videojuegos, ya bien desde su análisis como historias (narratología) o bien como juegos (ludología); para concluir con perspectivas más recientes de carácter interdisciplinar que permiten una comprensión de los mismos como medios de comunicación social.

4 Sin pretender adentrarse en la compleja definición de juego, en la segunda parte del primer capítulo se recogen teorizaciones de diversos autores sobre los elementos que lo componen, destacando las mecánicas de juego, las cuales definen los objetivos, reglas o elecciones posibles para el jugador. Para comprender el proceso de imbricación de los elementos narrativos y los lúdicos, el autor se apoya en la retórica de procesos de Ian 
Bogost (2007). Así, en los videojuegos, la persuasión, en vez de producirse mediante el uso de la palabra, la escritura o la imagen audiovisual, se articula mediante las reglas e interacciones simbólicas que guían las posibilidades de interacción del jugador. Para ejemplificar este modelo de acercamiento, se analiza el videojuego crítico McDonald's Videogame (Molleindustria, 2006), en el cual su sistema de reglas está diseñado para hacer descubrir al jugador que este tipo de negocios de carácter multinacional sólo pueden resultar muy rentables si se comenten una serie de tropelías sociales, políticas o medioambientales.

5 El autor aborda el concepto de los serious games desde su naturaleza híbrida como software de entretenimiento y educativo. Haciendo un repaso por las principales clasificaciones, plantea dos grandes grupos: los dirigidos a la persuasión en el terreno publicitario (advergames) o propagandístico, muchos de los cuales pretenden transmitir una determinada visión del mundo, por un lado, y por otro, los orientados a la adquisición de información y/o aprendizaje, estructurados en torno a objetivos pedagógicos, del ejercicio de ciertos comportamientos o relacionados con la actualidad informativa (newsgames). Asimismo, se aclara la diferencia entre los serious games y la gamificación, consistente, esta última, en trasladar técnicas propias de los juegos a la consecución de objetivos en contextos no lúdicos tales como el trabajo o la educación.

El segundo capítulo trata la utilización de los serious games por parte de las instituciones militares, el llamado militainment. En primer lugar, se realiza un breve recorrido histórico sobre el modo en que lo bélico ha estado siempre vinculado a juegos y contenidos de entretenimiento de la comunicación social. Siendo uno de los pasos más importantes dentro del videojuego como simulación bélica fue la incorporación del realismo que supuso el uso de la primera persona en los denominados first person shooters en los años noventa.

7 Dentro de las aplicaciones militaristas, destaca en el libro el caso de America's Army (2002), un videojuego capaz de competir con los grandes éxitos de la industria y que supuso un punto de inflexión en la estrategia del ejército estadounidense por revertir los bajos índices de reclutamiento y mejorar su aceptación social. Este videojuego refuerza el ideario ético militar estadounidense y así como la justificación de la violencia como modo de defender la libertad. Su representación visual se ajusta a sus necesidades comunicativas: el juego no incluye sangre ni heridas para poder ser catalogado como apto para su público objetivo, el adolescente. Se recogen diversos estudios que avalan la mejoría en la formación de los reclutas gracias a este tipo de juegos, no sólo en aspectos vinculados a la asimilación del funcionamiento interno del ejército o al campo de batalla, sino también los relacionados con la negociación y la diplomacia. Así, en juegos como Connect with Haji Kamal (Kinection \& World Warfighter, 2010), el jugador ha de saber convencer al líder de una tribu afgana para conseguir su colaboración para luchar contra los insurgentes.

8 Se analizan también los casos de Gran Bretaña y el -mucho más modesto- de España, en el cual se han desarrollado videojuegos vinculados a la labor humanitaria del ejército, obviándose los aspectos bélicos inherentes a las instituciones militares.

Un interesante apartado es el dedicado al militainment desde perspectivas que rompen la hegemónica ideológica de la industria del entretenimiento occidental, tal como la planteada por los países árabes, en los que se narra a modo de respuesta la visión de los conflictos desde su propia perspectiva. Juegos como Under Ash (bajo las cenizas) (Dar alFikr, 2001) y Under Siege (bajo el asedio) (Afkar Media, 2005) son first person shooters que 
recrean la historia reciente de Palestina en su enfrentamiento con la ocupación israelí durante la primera y segunda intifada, en los que el jugador debe, entre otras cosas, ayudar a escapar de las balas israelíes a los heridos o enfrentarse a tanques con piedras.

La capacidad de los serious games para el fomento y la concienciación en distintas causas sociales es analizada en el tercer capítulo. El autor narra cómo en sus inicios este tipo de uso se encontró con la incomprensión de quienes lo vinculaban a la tradicional frivolidad de los videojuegos de entretenimiento. Este capítulo se articula a través del análisis de diferentes juegos considerados destacables. El primero de ellos es Escape from Woomera (EFW Collective, 2003). Creado por activistas a partir de la modificación de un videojuego 3D comercial, tenía por objetivo denunciar las restricciones impuestas por el gobierno australiano para impedir que los medios de comunicación pudieran acceder al centro de detención de refugiados Woomera, El jugador, que encarna a un inmigrante, debe intentar escapar de dicho centro, correspondiéndose los distintos planes de fuga con intentos y hechos reales.

11 En Darfur is Dying (Take Action Games, 2006), videojuego catalogado como "de supervivencia", el jugador ha de atravesar un desierto hasta llegar a un pozo de agua y volver con él a su campamento mientras esquiva a la guerrilla. Las reglas y mecánica del juego sirven para ejemplificar cómo se conforma con ellas un determinado discurso, en este caso divulgando lo trágico de este tipo de catástrofes, pues no hay un final feliz en ninguna de sus resoluciones. Según la edad y sexo del personaje que se elija, se dispondrá de mayor o menor ventaja física para llevar a cabo la labor, así como se correrá mayor o menor riesgo de ser asesinado o violado.

Otros juegos analizados en esta línea son Food Force (Depend \& Playthree, 2005), del Programa Mundial de Alimentos de Naciones Unidas y Against all odds (United Nations Refugee Agency, 2005) de la oficina de Alto Comisionado de las Naciones Unidas para los Refugiados (ACNUR), el cual ha sido diseñado para jóvenes de entre 12 a 15 años con el fin de incluirse en el currículo académico dentro de las asignaturas de educación cívica. En el caso del juego Ayiti: the cost of live (Global Kids \& GameLab, 2006), desarrollado para UNICEF, el jugador ha de saber administrar una familia de Haití para que salga adelante. El mismo se acompaña de material para el profesorado, de forma que éste pueda ser utilizado en el discurso educativo en el aula.

13 También se analizan juegos cuyo objetivo es explicar los motivos y naturaleza de los conflictos internacionales, como el caso de Global Conflict : Palestine (SGI, 2007) o Global Confli t: Latin America (SGI, 2008), en los que el estudiante, en el papel de un periodista, ha de entrevistarse con las diferentes partes implicadas, para posteriormente redactar diferentes reportajes de carácter académico evaluable. En PaceMaker (Impact Games, 2007), en el que desde la perspectiva de la presidencia de Israel o Palestina, el jugador debe intentar conseguir la paz, obteniendo un equilibrio entre todos los sectores de opinión implicados, con la dificultad de que medidas éticamente correctas pueden ser no bien recibidas por ciertas partes e incrementar la tensión. Se recurre a noticias audiovisuales reales para reforzar la sensación de realismo. También se destaca Stop Disaster (Player-three, 2008), en el que el jugador ha de aprender a gestionar una catástrofe natural, evaluando riesgos, tipos de construcciones, alertas, simulacros, etc. salvando el mayor número de vidas posible.

El capítulo cuarto se dedica a los newsgames, los videojuegos consistentes en plasmar aspectos informativos de la actualidad. Frecuentemente, éstos se acompañan de una intención editorialista en la que el planteamiento y reglas del juego hacen posicionarse 
al jugador ideológicamente frente a lo representado. El autor expone dos grandes etapas. Una primera, que abarca de 2003 a 2006, en la que los juegos eran de producción modesta y generalmente vinculados a iniciativas puntuales con un contenido cercano a lo que supondrían en otros medios una viñeta o un sketch. Por su carácter editorialista, se analiza el juego, perteneciente a esta etapa, September 12th (Powerful Robots, 2003), un juego sencillo en el cual el jugador, en su intento de lanzar un misil que mate a terroristas, acaba generando más terroristas, surgidos de entre las víctimas. En la segunda etapa, de 2007 a 2011, se produjo un respaldo por parte de los medios tradicionales, quienes desarrollaron juegos que ilustraban, por ejemplo, las dificultades de la aduana estadounidense para evitar la entrada de alimentos contaminados, o lo problemático de obtener tarjetas de residencia en EEUU a partir de una reforma legislativa implantada por el gobierno.

Por último, se analiza el caso español, en los que juegos modestos abordan desde una perspectiva satírica la corrupción en el Partido Popular o el funcionamiento de los partidos políticos en campaña electoral.

En el quinto capítulo, de forma breve, se analizan los healthcare games, los videojuegos orientados al tratamiento y comprensión de enfermedades o a mejorar las condiciones físicas o mentales del jugador. De los años noventa, a modo de ejemplo, se analiza el juego Capitan Novolin (Raya System, 1992), encaminado a concienciar a jugadores diabéticos sobre las pautas a seguir para una alimentación saludable. A este título le siguieron otros para la concienciación contra el tabaco, para ayudar a convivir con el asma o el cáncer. Todo ello con el objetivo final de conseguir mejoras en la salud. Otro grupo destacado es el relacionado con la actividad lúdica física, tales como juegos desarrollados para Wii Fit (Nintendo, 2007), o Brain Training (Nintendo, 2005) dirigido al mantenimiento o mejora de la actividad cerebral.

El último, y muy breve, capítulo supone una reflexión final en la que se sugiere un futuro en el que los serious games podrían tener un mayor protagonismo, dada la expansión del medio, y debido a las expectativas que en ellos han depositado diversos educadores o profesionales del marketing, así como constituir una de las posibles salidas a la actual crisis del sector periodístico.

La obra, escrita con un lenguaje claro y pedagógico, supone un interesante acercamiento a las características y posibilidades de un ámbito en auge. El libro, quizás precisamente por el interés que puede suscitar, resulta breve. Puede echarse en falta el propio autor lo reconoce- una mayor profundización sobre ciertos aspectos abordados en él. No se analizan dos campos muy amplios e importantes de los seriuos games: educación y publicidad. El autor lo justifica al tratarse de temáticas mucho más populares y que gozan de una mayor disponibilidad de literatura en castellano. Por otro lado, el libro no responde -ni lo pretende- a la ambiciosa pregunta que le da título (¿pueden los videojuegos cambiar el mundo?). Desde una pretensión manifiestamente introductoria y descriptiva, se explicitan algunas de las ventajas de este tipo de videojuegos desde la prudencia académica, a partir de casos concretos, sin caer en posibles afirmaciones sustentadas en un optimismo no documentado. El libro, sin duda, constituye un buen lugar por el que empezar para quien esté interesado en la investigación de los serious games desde la perspectiva de las ciencias de la comunicación. 


\section{BIBLIOGRAFÍA}

Referencias bibliográficas

Bogost, I. Persuasive Games. The Expressive Power of Videogames. MIT Press, Cambridge, 2007

Videojuegos citados

Against all odds (United Nations Refugee Agency, 2005). Disponible en: http://

www.playagainstallodds.ca/

America's Army: Recon (United States Army, 2002). Disponible en: http://www.americasarmy.com/

Ayiti: the cost of live (Global Kids \& GameLab, 2006). Disponible en: https://ayiti.globalkids.org/ game/

Brain Training (Nintendo, 2005).

Capitan Novolin (Raya System, 1992).

Connect with Haji Kamal (Kinection \& World Warfighter, 2010). Disponible en : http:// www.worldwarfighter.com/hajikamal/

Darfur is Dying (Take Action Games, 2006). Disponible en : http://www.darfurisdying.com

Escape from Woomera (EFW Collective, 2003). Disponible en : http://www.ljudmila.org/

rselectparks/archive/escapefromwoomera/

Food Force (Depend \& Playthree, 2005).

Global Conflict: Latin America (SGI, 2008). Disponible en : http://globalconflicts.eu/

Global Conflict: Palestine (SGI, 2007). Disponible en : http://globalconflicts.eu/

McDonald's Videogame (Molleindustria, 2006). Disponible en : http://www.mcvideogame.com/

PaceMaker (Impact Games, 2007). Disponible en : http://www.peacemakergame.com/

September 12th (Powerful Robots, 2003). Disponible en : http://www.newsgaming.com/games/ index12.htm

Stop Disaster (Player-three, 2008). Disponible en : http://www.stopdisastersgame.org

Under Ash (Dar al-Fikr, 2001).

Under Siege (Afkar Media, 2005). Disponible en : http://www.underash.net/en_download.htm Wii Fit (Nintendo, 2007).

\section{AUTOR}

\section{ALFONSO BOULLÓN SABÍN}

Doctorando en Ciencias de la Comunicación

Sección de Ciencias de la Información

Universidad de La Laguna 